Z Rheumatol $2017 \cdot 76: 648$

DOI 10.1007/s00393-017-0365-6

๑) Springer Medizin Verlag GmbH 2017

\section{Deutsche $\mid$ RHEUMA-LIGA}

- gemeinsam mehr bewegen -
Herausgegeben im Auftrag

des Vorstandes

Rotraut Schmale-Grede (V.i.S. d.P.)

Präsidentin Deutsche Rheuma-Liga

Bundesverband e.V.

Maximilianstr. 14

53111 Bonn

T: 0228-76606-0

bv@rheuma-liga.de

www.rheuma-liga.de
Redaktion

Miliana Romic (V.i. S.d.P.)

Leiterin Presse- und

Öffentlichkeitsarbeit

Deutsche Rheuma-Liga

Bundesverband e.V.

Maximilianstr. 14

53111 Bonn

T: 0228-76606-11

bv.romic@rheuma-liga.de

www.rheuma-liga.de

\author{
Jürgen Clausen ${ }^{1} \cdot$ Roswitha Dietzel $^{2} \cdot$ Gabriele Armbrecht $^{2}$ \\ 'Deutsche-Rheuma-Liga Bundesverband e.V., Maximilianstraße 14, \\ 53111 Bonn \\ ${ }^{2}$ Zentrum für Muskel- und Knochenforschung, Charité - Campus \\ Benjamin Franklin, Hindenburgdamm 30, 12200 Berlin
}

\title{
Sarkopenie bei Patienten mit rheumatischen Erkrankungen
}

Der Begriff „Sarkopenie“ bezeichnet den mit fortschreitendem Alter zunehmenden Muskelmasse- und Muskelfunktionsabbau. Dieser Abbau führt $\mathrm{zu}$ deutlichen Beeinträchtigungen bei der Verrichtung normaler Tätigkeiten im Alltag und erhöht das Risiko für Stürze und daraus resultierende Frakturen, die wiederum Immobilität und Verlust der Unabhängigkeit nach sich ziehen können. Der Abbau beider Komponenten ist Teil des natürlichen Alterungsprozesses, wird aber als Sarkopenie definiert, sobald ein bestimmter Schwellenwert unterschritten wird. Derzeit gibt es wenigstens vier unterschiedliche Definitionen der Sarkopenie [1], was unter anderem die Vergleichbarkeit von Studienergebnissen erschwert. Ein einheitlicher Standard ist hier anzustreben.

Nach Schätzungen sind ungefähr $45 \%$ der älteren US Bürger von Sarkopenie betroffen [2], aber auch junge Menschen mit chronischen Erkrankungen können betroffen sein [3]. Aufgrund der stetig älter werdenden $\mathrm{Ge}$ sellschaft ist die Sarkopenie als großes Problem für das Gesundheitswesen erkannt worden, das in Zukunft bedeutende klinische, ökonomische und soziologische Konsequenzen nach sich ziehen wird [4]. Darüber hinaus ist die Sarkopenie für Betroffene mit rheumatischen Erkrankungen von besonderer Bedeutung, da von den bisher erkannten Risikofaktoren zur Entwicklung einer Sarkopenie zwei Risikofaktoren für Rheumabetroffene besonders kritisch sind: 1. Mangelnde Bewegung und 2. Chronische Entzündungen. Somit ist bei Menschen mit entzündlich rheumatischen Erkrankungen die Sarkopenie nicht nur ein geriatrisches Problem, sondern sie kann bei diesem Personenkreis bereits in früheren Lebensjahren auftreten.

Um dieser besonderen $\mathrm{Si}$ tuation Rechnung $\mathrm{zu}$ tragen, hat die Deutsche Rheuma-Liga 2016 ihre Förderausschreibung mit dem Thema „Sarkopenie bei rheumatischen Erkrankungen“ ausgeschrieben. Ziel dieser Ausschreibung war es, Forschung anzuregen, die die Entstehung, das Voranschreiten, die Prävention und die Therapie der Sarkopenie untersucht. Neun Anträge sind bei der Deutschen Rheuma-Liga eingegangen, von denen letztlich das Projekt von Frau Dr. Gabriele Armbrecht und Herrn Prof. Dr. Buttgereit, beide Charité Berlin, zur Förderung ausgewählt wurde.

Im Projekt mit dem Titel „Sarkopenie bei Patienten mit rheumatischen Erkrankungen“ soll untersucht werden, wie weit die Sarkopenie bei Patienten mit rheumatoider Arthritis (RA) verbreitet ist. Unter Anwendung dreier verschiedener SarkopenieDefinitionen wird im Rahmen einer Querschnittsstudie erhoben, bei wie vielen RA-Patienten eine Sarkopenie vorliegt. Die Güte und Praktikabilität der drei Definitionen werden dabei miteinander verglichen. In einem zweiten Schritt werden die erhobenen Daten der RA Patienten mit Daten von Kontrollen ohne RA verglichen (Fall-Kontrollstudie). Untersucht werden Muskelmasse, Muskelfunktion, körperliche Aktivität, Lebensqualität und Laborparameter.

Die Ergebnisse sollen außerdem dazu beitragen, das komplexe Phänomen der Sarkopenie besser zu verstehen und Risikofaktoren $\mathrm{zu}$ identifizieren, um zukünftige Ansätze für eine verbesserte Diagnostik zu liefern. Darüber hinaus sollen präventive Trainingsinterventionen für Rheumapatienten aus den Ergebnissen abgeleitet werden. Angeschlossen an das Projekt ist eine prospektive Sturzbefragung über 12 Monate (gefördert durch die Stiftung Wolfgang Schulze) zur Ermittlung der Sturzinzidenz und von Risikofaktoren.

\section{Literatur}

1. Peterson SJ, Braunschweig CA (2016) Prevalence of Sarcopenia and Associated Outcomes in the Clinical Setting. Nutr Clin Pract 31(1):40-48

2. Janssen I, Shepard DS, Katzmarzyk PT, Roubenoff R (2004) The healthcare costs of sarcopenia in the United States. J Am Geriatr Soc 52(1):80-85

3. Cruz-Jentoft AJ, Baeyens JP, Bauer JM, Boirie Y, Cederholm T, Landi F, Martin FC, Michel JP, Rolland Y, Schneider SM, Topinková E, Vandewoude M, Zamboni M (2010) European Working Group on Sarcopenia in Older People. Sarcopenia: European consensus on definition and diagnosis: Report of the European Working Group on Sarcopenia in Older People. Age Ageing 39(4):412-423

4. Beaudart $C$, Rizzoli $R$, Bruyère $O$, Reginster JY, Sarcopenia BE (2014) burden and challenges for public health. Arch Public Health 18; 72(1):45 